\title{
Cutaneous Changes During Pregnancy
}

\author{
Heba Ahmad Anwar Mahmoud ${ }^{1}$, Salah Mohamed Rasheed ${ }^{2}$, RehamEzz El-Dawla \\ El Sharkawy ${ }^{I}$ \\ 1. Departments of Dermatology, Venereology and Andrology, Sohag University \\ 2. Department of Obstetrics and Gynaecology, Sohag University
}

\section{Abstract}

Background:There are several changes which affect females' skin that occur during pregnancy. These changes, although considered, physiological are quite distressing to many women. Discussion with the pregnant woman about the nature of her skin condition and the possible fetal risks associated with it is imperative.

Aim of the work: This study aimed to evaluate the pattern of dermatological manifestations which affect pregnant women in our environment with different socio-economic levels.

Patients and Methods: This study was a cross- sectional design. It included 600 pregnant women who attended Dermatology, Venereology, Andrology and Antenatal care clinics. Local examination included morphology of skin changes (monomorphic or polymorphic), type of lesion (primary or secondary), distribution of skin disease (localized or generalized, unilateral or bilateral) and site of the lesion.

Results: The most common physiological skin changes were pigmentary changes $(98 \%)$ followed by striae distensae (76\%), vascular changes (27.3\%), hair changes (20.2\%), glandular changes $(19 \%)$ and nail changes $(2 \%)$. As regarding pigmentary changes; lineanigra $(86.6 \%)$, secondary areola $(74.3 \%)$ and melasma $(44 \%)$ were most common pigmentary changes in pregnant women.Scabies was the most common associated dermatological disorder $(2.7 \%)$ of pregnant women followed by acute urticaria $(1.3 \%)$, polymorphic light eruption (1.3\%) and molluscum contagiosum (0.3\%) of patients.Pregnancy-induced dermatoses were found in $67(11.3 \%)$ of pregnant women. Atopic eruption of pregnancy (44.8\%) was the most common condition [i.e. includes eczema (23.9\%), prurigo (14.9\%) and pruritis folliculitis (6\%)]; polymorphic eruptions (44.8\%), intrahepatic cholestasis of pregnancy $(6 \%)$ and pemphigoid gestationis $(4.5 \%)$ of pregnant women.

Conclusion: Pregnancy is a unique physio-logical state characterized by metabolic, immunologic, and hormonal readjustments. These make a pregnant woman vulnerable to all dermatoses occurring in the non-pregnant state and also to certain eruptions related to the physiologic burden of gestation.

Keywords: retro peritoneal neuroblastoma, children, surgery, chemotherapy, radiotherapy.

\section{Introduction}

There are several changes which affect females' skin that occur during pregnancy. These changes, although considered, physiological are quite distressing to many women (1). Discussion with the pregnant woman about the nature of her skin condition and the possible fetal risks associated with it is imperative $(2,3)$.

During pregnancy, immunologic, metabolic, endocrine and vascular changes occur, which make the woman susceptible to changes of the skin and appendages. Recognition of these changes is important for correct classification and appropriate treatment when necessary (4-6).
The cutaneous changes during pregnancy can be classified in to 3 types: physiologic skin changes in pregnancy, dermatoses and cutaneous lesions affected by pregnancy, and dermatoses only occurring in pregnancy, such as intrahepatic cholestasis of pregnancy and specific dermatoses of pregnancy $(2,7$, 8).

Pregnancy-related skin changes are most likely caused by the hormonal changes associated with pregnancy. They have also been called physiologic skin changes or skin changes of endo-crine origin e.g. chloasma, hirsutism, postpartum telogen effluvium, postpartum male-pattern alopecia, Striae, 
SOHAG MEDICAL JOURNAL

Spider telangiectasia and palmer erythema(9-12). Hyperpigmentation isacommon feature of pregnancy and may be seen in up to $90 \%$ of pregnant women $(1,3,5,6)$.

The physiology of hyperpigmen-tation and chloasma may be related to elevated serum levels of melanocyte-stimulating hormone, estrogen and possibly progesterone (13). Mild generalized hyperpigmentation is usually seen with accentuation of normally hyperpigmented areas such as the areola, nipples, genital skin, axillae and inner thighs $(1,8,11)$.

Cutaneous disorders and lesions that could occur during pregnancy such as atopic dermatitis, psoriasis, acne vulgaris, urticaria, fungal, herpes virus, condyloma acuminatum, systemic sclerosis, dermatomyositis, polymyositis, rheumatoid arthritis, leprosy, pemphigus vulgaris, pyogenic granuloma, bowenoid papulosis, desmoid tumor, neurofibroma, melanocytic nevus and melanoma $(2,3,14,15)$.

Atopic dermatitis is more likely to worsen during pregnancy. The exacerbation is partly attributed to the pruritus of pregnancy, chronic plaque psoriasis is the most common type of psoriasis to develop or worsen in pregnancy $(2,7,12,16,17)$.

Dermatoses of pregnancy include conditions that occur exclusively during pregnancy, such as intrahepatic cholestasis of pregnancy, and specific dermatoses of pregnancy as Herpes gestosis, Pruritic urticarial papules, plaques of pregnancy, Prurigo of pregnancy and Pruritic folliculitis of pregnancy $(11,18,19)$.

Intrahepatic cholestasis of pregnancy is seen in the $3^{\text {rd }}$ trimester of pregnancy in $70 \%$ of cases and is due to a mild form of intrahepatic bile secretory dysfunction $(12,16)$.

Herpes gestosis is characterized by pruritic and/or urticarial plaques containing vesicles beginning on the abdomen and can extend to become a generalized bullous eruption(20).
Cutaneous Changes During Pregnancy

Heba Ahmad Anwar Mahmoud
Aim of the work:

This study aimed to evaluate the pattern of dermatological manifesta-tions which affect pregnant women in our environment with different socioeconomic levels.

\section{Patients and Methods:}

This study was a cross- sectional design. It included 600 pregnant women who attended Dermatology, Venereology, Andrology and Antenatal care clinics. The study was approved by the Research and Ethical Comm-ittees at Sohag Faculty of Medicine, Sohag University. Informed written consent was obtained from all pregnant women before inclusion in the study.

All pregnant women were subjected to the following:

History taking:

1. Personal history: (name, age, residence, occupation, special ha-bits and socioeconomic level).

2. Menstrual and obstetric history:(gestational age, parity, history of twin pregnancy).

3. History of the present illness: time of skin changes $\left(1^{\text {st }}, 2^{\text {nd }}\right.$ or $3^{\text {rd }}$ trimester $)$, type of skin changes in each of the three trimesters, onset, course, duration, sequence of sym-ptoms and exacerbating factors.

4. History of other conditions: associated symptoms, other skin diseases, chronic diseases (DM, hypertension etc).

5. Medical history: hormonal contraception, dermatological and non dermatological medications.

6. Past history: previous similar skin changes in previous pregnancy, postpartum course (remission or exacerbation), last delivery (normal or ceserian section).

7. Family history of similar conditions.

\section{Examination:}

1. General examination included vital signs (blood pressure, pulse, and temperature), mucous membranes, hair and nail.

2. Local examination included morphology of skin changes 
SOHAG MEDICAL JOURNAL

Vol. 22 No.3 October 2018

(monomorphic or polymorphic), type of lesion (primary or secondary), distribution of skin disease (localized or generalized, unilateral or bilateral) and site of the lesion.

\section{Statistical analysis:}

- Statistical package for social sciences (IBM-SPSS), version 24 IBM- Chicago, USA (May 2016) was used for statistical data analysis.

- Data expressed as mean, standard deviation (SD), number and percentage. Mean and standard

\section{Results}

deviation were used as descriptive value for quantitative data, while number and percentage were used to describe qualitative data.

- Pearson Chi square was used to compare percentages of qualitative data, and Fisher's Exact test was used for non parametric data.

- A P-value less than 0.05 was considered as being statistically significant.

This study included 600 pregnant women, aged (18-45) years with nearly half of them between (18-25) years of age (young adults) and around 40\% ranged from (26-40) years. Only a minority of pregnant women aged more than 40 years $(11.8 \%) .40 \%$ of pregnant women were primigravida, while $55 \%$ multigravida $(2-4)$ and $5 \%$ were grand multigravida (>4) (Table 1).

Table 1. Demographic data of the study group.

\begin{tabular}{|c|c|c|c|}
\hline & $N_{0}=600$ & No. & Percent $\%$ \\
\hline \multirow[t]{3}{*}{ Age } & Young Adults (18-25 years) & 281 & $46.8 \%$ \\
\hline & Adults (26-40 years) & 248 & $41.3 \%$ \\
\hline & Middle age (>40 years) & 71 & $11.8 \%$ \\
\hline \multirow[t]{3}{*}{ Residence } & Rural & 160 & $26.7 \%$ \\
\hline & Urban & 392 & $65.3 \%$ \\
\hline & Suburban & 48 & $8.0 \%$ \\
\hline \multirow{2}{*}{ Occupation } & Housewife & 359 & $59.8 \%$ \\
\hline & Employed & 241 & $40.2 \%$ \\
\hline \multirow[t]{2}{*}{ Special habits } & Smoker & 29 & $4.8 \%$ \\
\hline & Non smoker & 571 & $95.2 \%$ \\
\hline \multirow[t]{3}{*}{ Sociocconomic level } & High & 23 & $3.8 \%$ \\
\hline & Middle & 362 & $60.3 \%$ \\
\hline & Low & 215 & $35.8 \%$ \\
\hline \multirow[t]{6}{*}{ Gravidity } & 1 & 244 & $40.7 \%$ \\
\hline & 2 & 185 & $30.8 \%$ \\
\hline & 3 & 87 & $14.5 \%$ \\
\hline & 4 & 55 & $9.2 \%$ \\
\hline & 5 & 18 & $3.0 \%$ \\
\hline & 6 & 11 & $1.8 \%$ \\
\hline \multicolumn{2}{|l|}{ Total } & 600 & $100.0 \%$ \\
\hline
\end{tabular}

The majority of pregnant women were normotensive with normal heart rate and body temperature. However, diastolic blood pressure (DBP) was elevated in some pregnant women with a mean \pm SD $(77.39 \pm 14.297 \mathrm{~mm} \mathrm{Hg})$. Pulse was normal, with a mean \pm SD $(73.77 \pm 8.926 \mathrm{~b} / \mathrm{m})$. Body temperature was normal with a mean $\pm \mathrm{SD}$ $\left(37.07 \pm 0.580^{\circ} \mathrm{C}\right)$.

This study reported that $60.3 \%$ of pregnant women were in the second trimester of pregnancy $(60 \%)$, while only $21 \%$ of pregnant women were in the third trimester and $19 \%$ were in the first trimester. There is a statistically significant increase in dermatological manifestations in the second trimester compared to both first and third trimester ( $\mathrm{P}$ value $<0.001$ ). Regarding the duration of dermatological complaints, 490 $(80.7 \%)$ of pregnant woman had a short duration of less than 2 months, while only $110(18.3 \%)$ cases had disease duration of more than 2 months.

In this study, the most common physiological skin changes were pigmentary changes 
(98\%) followed by striae distensae (76\%), vascular changes $(27.3 \%)$, hair changes $(20.2 \%)$, glandular changes (19\%) and nail changes (2\%). As regarding pigmentary changes; lineanigra (86.6\%), secondary areola $(74.3 \%)$ and melasma $(44 \%)$ were most common pigmentary changes in pregnant women (Table 2).

Table 2. Distribution of pregnant women according to physiological changes in pregnancy $(n=600)$.

\begin{tabular}{|l|l|c|c|}
\hline Physiological changes & & No.of cases & Percent\% \\
\hline 1-Pigmentation & & $\mathbf{5 8 8}$ & $\mathbf{9 8 \%}$ \\
\hline & a-Linea nigra & $\mathbf{5 2 0}$ & $\mathbf{8 6 . 6 \%}$ \\
\hline & b-Secondary areola & $\mathbf{4 4 6}$ & $\mathbf{7 4 . 3 \%}$ \\
\hline & c-Melasma & $\mathbf{2 6 4}$ & $\mathbf{4 4 \%}$ \\
\hline & d-Naevi darkening & $\mathbf{8 7}$ & $\mathbf{1 4 . 5 \%}$ \\
\hline & e-Pigmentary demarcation line & $\mathbf{6 6}$ & $\mathbf{1 1 \%}$ \\
\hline 2-Striae distensae & & $\mathbf{4 5 6}$ & $\mathbf{7 6 \%}$ \\
\hline & & $\mathbf{1 1 4}$ & $\mathbf{1 9 \%}$ \\
\hline & a-Acne & $\mathbf{7 5}$ & $\mathbf{1 2 . 5 \%}$ \\
\hline & b-Montogmery'- stubercule & $\mathbf{3 1}$ & $\mathbf{5 . 1 \%}$ \\
\hline 4-Vascular changes & c-Miliaria & $\mathbf{8}$ & $\mathbf{1 . 4 \%}$ \\
\hline & & $\mathbf{1 6 4}$ & $\mathbf{2 7 . 3 \%}$ \\
\hline & a-Nonpitting edema of feet & $\mathbf{7 3}$ & $\mathbf{1 2 . 2 \%}$ \\
\hline & b-Palmar erythema & $\mathbf{1 9}$ & $\mathbf{3 . 1 \%}$ \\
\hline & c-Spider telangectesia & $\mathbf{6 0}$ & $\mathbf{1 0 \%}$ \\
\hline 5-Nail Changes & d-Varicosities of legs & $\mathbf{2 5}$ & $\mathbf{4 . 2 \%}$ \\
\hline 6-Hair changes & & $\mathbf{2 1}$ & $\mathbf{2 0 . 3} \%$ \\
\hline
\end{tabular}

Scabies was the most common associated dermatological disorder (2.7\%) of pregnant women followed by acute urticaria (1.3\%), polymorphic light eruption $(1.3 \%)$ and molluscum contagiosum $(0.3 \%)$ of patients (Figure 1$)$.

Pregnancy-induced dermatoses were found in 67 (11.3\%) of pregnant women. Atopic eruption of pregnancy $(44.8 \%)$ was the most common condition [i.e. includes eczema (23.9\%), prurigo (14.9\%) and pruritis folliculitis (6\%)]; polymorphic eruptions $(44.8 \%)$, intrahepatic cholestasis of pregnancy $(6 \%)$ and pemphigoid gestationis $(4.5 \%)$ of pregnant women (Figure 2 ).

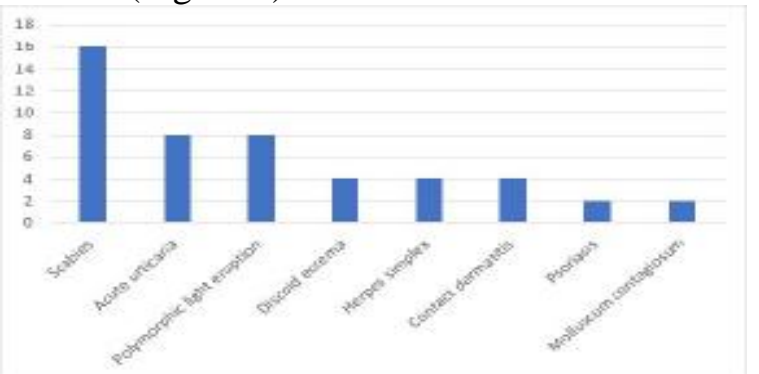

Figure (1). Distribution of pregnant women according to associated dermatological disorders.

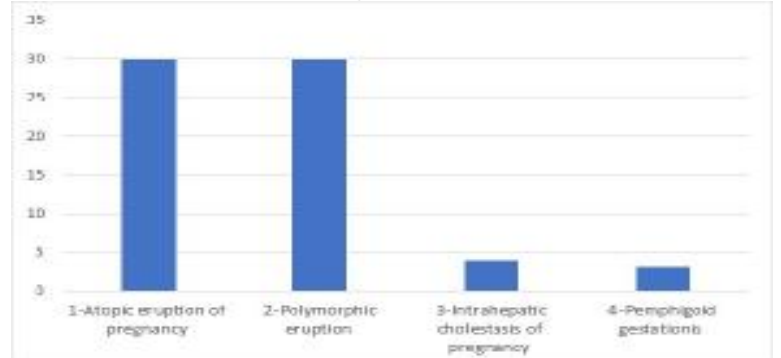

Figure (2). Distribution of subjects according to specific dermatoses of pregnancy 


\section{Mucous membrane, hair and nail changes of the study group:}

Oral candidiasis $(5.3 \%)$ was the most common mucous membrane affection of pregnant wonen, followed by parulis $(4.0 \%)$, petechiae $(3.5 \%)$ and gingivitis $(1.5 \%)$. $91(15.2 \%)$ of pregnant wonen showed increases hair loss and $31(5.2 \%)$ of pregnant wonen showed improved hair growth. Most common nail changes of pregnant wonen were brittle nails $(0.8 \%)$ followed by transverse grooving $(0.5 \%)$ (Table 3$)$.

Table 3. Mucous membrane, hair and nail changes of the study group.

\begin{tabular}{|l|l|c|c|}
\hline \multicolumn{2}{|c|}{} & No.of cases & Percent \\
\hline \multirow{4}{*}{ Oral/vaginal mucous membrane } & Oral Candidiasis & $\mathbf{3 2}$ & $\mathbf{5 . 3 \%}$ \\
\cline { 2 - 4 } & Parulis & $\mathbf{2 4}$ & $\mathbf{4 \%}$ \\
\cline { 2 - 4 } & Fissured tongue & $\mathbf{6}$ & $\mathbf{1 \%}$ \\
\cline { 2 - 4 } & Mucocele & $\mathbf{6}$ & $\mathbf{1 \%}$ \\
\cline { 2 - 4 } & Petechiae & $\mathbf{1 5}$ & $\mathbf{3 . 5 \%}$ \\
\cline { 2 - 4 } & Gingivitis & $\mathbf{1 4}$ & $\mathbf{1 . 5 \%}$ \\
\hline \multirow{4}{*}{ Hair changes } & Increased hair loss & $\mathbf{9 1}$ & $\mathbf{1 5 . 2 \%}$ \\
\cline { 2 - 4 } & Improved hair growth & $\mathbf{3 1}$ & $\mathbf{5 . 2 \%}$ \\
\hline Nail & Brittle nail & $\mathbf{8}$ & $\mathbf{1 . 3 \%}$ \\
\cline { 2 - 4 } & Transeverse grooving & $\mathbf{4}$ & $\mathbf{0 . 7 \%}$ \\
\hline
\end{tabular}

Distribution of the physiological skin changes of pregnant women according to trimester:

In $1^{\text {st }}$ trimester, the most common physiological skin changes of pregnant woman were pigmentary changes $(90.1 \%)$, hair changes $(49.1 \%)$, glandular changes (35.7\%), vascular changes $(29.5 \%)$ and mucosal changes $(5.4 \%)$.In $2^{\text {nd }}$ trimester, the most common physiological skin changes of pregnant woman were pigmentary changes $(99.7 \%)$, connective tissue changes $(92.5 \%)$, glandular changes $(16.3 \%)$, vascular changes $(12.4 \%)$, hair changes $(9.9 \%)$, and mucosal changes $(7.7 \%)$. In $3^{\text {rd }}$ trimester, the most common physiological skin changes of pregnant woman were pigmentary changes $(100 \%)$, connective tissue changes (96\%), vascular changes $(68.3 \%)$, mucosal changes $(54.8 \%)$ and hair changes $(24.6 \%)$.

Distribution of the specific dermatoses of pregnant women according to trimester.

No specific dermatoses were found in $1^{\text {st }}$ trimester. The most common specific dermatoses in $2^{\text {nd }}$ trimester were atopic eruption of pregnancy $(6.1 \%)$ include eczema (3.3\%) and prurigo (1.9\%). The most common specific dermatoses in 3rd trimester were polymorphic eruption (23.8\%), atopic eruption of pregnancy (6.3\%), intrahepatic cholestasis of pregnancy (3.2\%) and pemphigoid gestationis (1.6\%). There is statistically significant increase atopic eruption of pregnancy in $2^{\text {nd }}$ trimester ( $\mathrm{P}$ value $<0.001)$. There is statistically significant increase polymorphic eruption of pregnancy in $2^{\text {nd }}$ trimester ( $\mathrm{P}$ value $<0.001$ ).

Relation between age groups of pregnant women and skin changes during pregnancy.

The majority of pregnant women were under 40 years of age and 71 pregnant women were over 40 years. The associated skin lesions were more prevalent with older patients, but with non significant difference $(\mathrm{P}=0.207)$. Specific lesions was significantly higher among patients with age groups 26-40 and over 40 years, compared to those below 26 years $(\mathrm{P}<0.001)$ (Table 4$)$.

Table 4. Relation between age groups of pregnant women and skin changes during pregnancy.

\begin{tabular}{|l|c|c|c|c|}
\hline \multirow{2}{*}{ Skin changes } & \multicolumn{3}{|c|}{ Age group of pregnant women } & \multirow{2}{*}{ P value } \\
\cline { 2 - 4 } & $\mathbf{1 8 - 2 5}(\mathbf{N}=281)$ & $26-40(\mathbf{N}=\mathbf{2 4 8})$ & $>40(\mathrm{~N}=71)$ & \\
\hline Physiological & $\mathbf{2 6 9}(100 \%)$ & $\mathbf{2 4 8}(100 \%)$ & $\mathbf{7 1}(100 \%)$ & 1.000 \\
\hline Associated & $18(6.4 \%)$ & $21(8.5 \%)$ & $9(12.7 \%)$ & 0.207 \\
\hline Specific & $12(4.3 \%)$ & $\mathbf{4 3}(17.3 \%)$ & $12(16.9 \%)$ & $<0.001$ \\
\hline
\end{tabular}




\section{Discussion}

Pregnancy is associated with significant cutaneous changes, which may range from physiological changes of skin to common skin diseases occurring coincidently with pregnancy, to eruptions seen only during pregnancy or postpartum period. Complex endocrinologic, immunologic, metabolic and vascular changes associated with pregnancy influence the skin in various ways(21).

Physiological cutaneous changes may be seen in almost all the pregnant females; which include changes in pigmentation, vascularsystem, striaedistensae and endocrine function, as well as, changes in hair and nails(2, 7).

In this study, pigmentary changes were seen in 588 (98\%) of pregnant women, of which lineanigra was the most common, seen in $520(86.6 \%)$ of pregnant women followed by secondary areola seen in $446(74 \%)$ of pregnant women. Martin and LealKhouri(22)reported that pigmentary alteration was seen in up to $90 \%$ of pregnant women, which is comparable to this study. Kumariet al(23)reported lineanigra, and secondary areola in (91.4\%) and $(78.4 \%)$ of their cases respectively which also matched withour study. In study by Panicker et al(24)pigmentary changes were seen in $(65.27 \%)$ of the pregnant women during the second trimester and in $(99.5 \%)$ of the pregnant women during the third trimester.

Melasma was seen in 264 (44\%) of pregnant women in this study. Consistent with our study Muzaffar et $\boldsymbol{a l}(25)$ found that melasma to be present in $(46 \%)$ of their pregnant women. In the contrary $\boldsymbol{R} \boldsymbol{a j} \boldsymbol{e t}$ $\boldsymbol{a l}(26)$ observed melasma in $(8.5 \%)$ of pregnant women and Kumari et al(23) reported melasma in $(2.5 \%)$ of pregnant women.

This difference may be due to pigmentary changes are more discernible in fair-skinned individuals. The disorder is known to be aggravated by ingestion of contraceptive pills and exposure tosunlight (27).

Kroumpouzo and Cohen(27)mention that striae distensae develop in up to $90 \%$ of women during the 6 th and 7 th month of pregnancy. Striae are mainly due to physical factors, stretching secondary to increase in the abdominal girth plays a major role. In this studystriae were seen in $456(76 \%)$ of pregnant women matched with this result Raj et al(26)., 1992 found striaedistensae in $(75 \%)$ of pregnant women.

Vascular changes in pregnancy result from distension, instability and proliferation of vessels during pregnancy. In the current study non pittingedema was seen in 73(12.2\%), varicosities of legs was seen in 25(4.2\%), palmar erythema was observed in $19(3.1 \%)$, whereas spider angiomas occurred in 60 (10\%).

Muzaffar et al(25) reported slightly increased prevalence of palmer erythema and spider nevi in their study (12\%) and (37\%) respectively. Raj et al(26)reported moderately increased prevalence of palmer erythemain their study (33.3).

These vascular changes seen during pregnancy are related to persistently raised levels of estrogen. The placenta is a rich source of basic fibroblast growth factor, a very active angiogenic factor in pregnancy(3).

In this study increased acne vulgaris reported in $75(12.5 \%)$ of pregnant women.Panicker et al(24)reported that $(1.3 \%)$ of pregnant women were showed acne vulgaris. The increased activity of sebaceous glands could be the cause for these diseases. Two factors that make acne more prevalent with pregnancy are the hormone, progesterone, and changes in hydration. Progesterone is secreted by 
the ovary in the first trimester, after that it is secreted by the placenta. But because progesterone is more androgenic than estrogen, it also causes increased sebum production and blockage of the pore opening which will lead to more acne(28), enlargement of glands on the areola (Montgomery's gland or tubercles) was observed in 31 (5.1) \%. Increased appearance of Montgomery's tubercles is well-known during pregnancy in 30$50 \%$ of pregnant women (22).

In this study, pregnancy gingi-vitis was seen in $14(1.5 \%)$ of the pregnant women. Muzzaffar et al(25)reported moderately increased preva-lence of gingival edema and redness $(16.4 \%)$.

Nail changes such as brittleness, subungual hyperkeratosis, onycholysis and leuconychia have been reported during pregnancy (27).

In this study, most common nail changes are transverse grooves and brittleness of nails which are observed in $12(2 \%)$ of the pregnant women.

In our study, $31(5.2 \%)$ of patients noticed improvement and lengthening of scalp hair, and $91(15.2 \%)$ reported increased hair loss.

In the study by Kumari et al(23) $2 \%$ gave a history of increased hair loss and only $(0.5 \%)$ patients noticed lengthening and improvement of their scalp hair, whereas (97.4\%) gave history of no change in hair density.

The present study reported that common infections were oral candidiasis 32 (5.3\%), scabies 16 $(2.6 \%)$, herpes infection $4(0.6 \%)$, and molluscum contagiosum $2(0.3 \%)$.

Panicker et al(24) found that vulvovaginal candidiasis was in $(21 \%)$ of pregnant women, dermatophytosis in $(21.6 \%)$ of cases, scabies in $(2.8 \%)$, pityriasis versicolor in $(6.16 \%)$ of cases, and molluscum contagiosum in $(0.3 \%)$ of the pregnant women.

An increased frequency of infection is common during pregnancy and is probably related to low cellular immunity. Reduced cell-mediated immunity means that viral warts and herpes simplex virus infections are more common in pregnancy and more severe, and can be transmitted to the baby during childbirth(29).

In this study, miliaria was reported in 8 $(1.4 \%)$ of pregnant women. It may be related to increased eccrine sweating and warm climate(2).

It has been noted that pregnancy causes eccrine gland hyperactivity; subsequently patients may have miliaria and hyperhidrosis $(3,24)$.

In the study, psoriasis vulgaris was found in $2(0.3 \%)$ pregnant women. There was no change in the activity of the disease during pregnancy. Panicker et al(24) reported that (1\%) pregnant women were showed psoriasis.

Pregnancy-specific skin derma-toses include an ill-defined, heterogeneous group of pruritic skin eruptions, which are seen only in pregnancy. The most rationalized classification was proposed by Ambros-Rudolph et $\boldsymbol{a l}(21)$ which is as follows: 1) atopic eruption of pregnancy (AEP) including a- eczema b-Prurigo; c- Pruritic folliculitis; 2) polymorphic eruption of pregnancy; 3) pemphigoid gestationis; and 4) intrahepatic cholestasis of pregnancy, described as specific dermatoses of pregnancy.

AEP is the most common pregnancyinduced dermatosis in the current study and observed in 30(44.8\%). AEP is a benign pruritic disorder of pregnancy, which includes eczematous and/or papular lesions in patients with an atopic diathesis after exclusion of the other dermatoses of pregnancy (21).

In the present study, eczema was observed in 16 (23.9\%) females. The eruption was seen more commonly in primigravida and skin lesions started during early pregnancy in first and second trimester. 
In our study, prurigo of pregnancy was seen in $10(14.9 \%)$ patients. also called as Besnier's prurigo, in contrast with us; Ambros-Rudoph et al(21)reported prurigo of pregnancy in $(9.4 \%),(7 \%)$ and $(0.8 \%)$ respectively.

Pruritic folliculitis of pregnancy was seen in $4(6 \%)$ patients in our study. This finding is consistent with the study done by Puri and Puri 2013 who reported in $(5.0 \%)$ of patient and in $(0.2 \%)$ of patient, commonly seen in the second and third trimester of pregnancy.

In the present study, PUPP was reported in $30(44.8 \%)$ patients. This is in contrast to the results quoted by Puri and Pooni(30) (62\%).Pemphigoid gestationis (PG) was observed in this study in $3(4.5 \%)$ patient. It is similar to those encountered byPuri and Pooni(30).

Intrahepatic cholestasis of pregnancy or pruritus gravidarum was seen in this study in $32(5.3 \%)$ of pregnant women. Results are similar to that of Puri and Pooni(30).

\section{Conclusion:}

Pregnancy is a unique physio-logical state characterized by metabolic, immunologic, and hormonal readjustments. These make a pregnant woman vulnerable to all dermatoses occurring in the non-pregnant state and also to certain eruptions related to the physiologic burden of gestation.

\section{References:}

1. Tunzi M, Gray GR. Common skin conditions during pregnancy. American family physician. 2007;75(2):211-8.

2. Ciechanowicz P, Sikora M, Taradaj K, Ruta A, Rakowska A, Kociszewska-Najman B, et al. Skin changes during pregnancy. Is that an important issue for pregnant women? Ginekologia polska. 2018;89(8):449-52.

3. Tyler KH. Physiological skin changes during pregnancy. Clinical obstetrics and gynecology. 2015;58(1):119-24.

4. Wong RC, Ellis CN. Physiologic skin changes in pregnancy. Journal of the American Academy of Dermatology. 1984;10(6):929-40.

5. Van Onselen J. Skin changes during pregnancy. Part 1. The journal of family health care. 2012;22(6):2830.

6. van Onselen J. Skin changes during pregnancy. Part 2: rash decision? The journal of family health care. 2013;23(1):14, 6-7.

7. Hassan I, Bashir S, Taing S. A clinical study of the skin changes in pregnancy in kashmir valley of north India: a hospital based study. Indian journal of dermatology. 2015;60(1):28-32.

8. Holmes RC, Black MM. The specific dermatoses of pregnancy: a reappraisal with special empha-sis on a proposed simplified clinical classification. Clinical and experimental dermatology. 1982;7(1):65-73.

9. Schmutz JL. [Physiological skin changes during pregnancy]. Presse medicale. 2003;32(38):1806-8.

10.Szamkolowicz G, Mierzecki A, Zaluga E. [Physiological skin changes during pregnancy]. Ginekologia polska. 2005;76(11):926-30.

11.Zerouali A, Zaraa I, Trojjet S, El Euch D, Azeiez MI, Mokni M, et al. [Physiologic skin changes in pregnancy]. Presse medicale. 2011;40(1 Pt 1):e17-21.

12.Wade TR, Wade SL, Jones HE. Skin changes and diseases associated with pregnancy. Obstetrics and gynecology. 1978;52(2):233-42.

13.Snell RS. The Pigmentary Changes Occurring in the Breast Skin during Pregnancy and Following Estrogen Treatment. The Journal of investigative dermatology. 1964;43:181-6. 
14.Wong RC, Ellis CN. Physiologic skin changes in pregnancy. Seminars in dermatology. 1989;8(1):7-11.

15.Hasan KM, Manyonda IT, Ng FS, Singer DR, Antonios TF. Skin capillary density changes in normal pregnancy and pre-eclampsia. Journal of hyper-tension. 2002;20(12):2439-43.

16.Kaplan RP. Skin changes and diseases associated with pregnancy. Obstetrics and gynecology. 1979;54(2):268.

17.Boyd AS, Morris LF, Phillips CM, Menter MA. Psoriasis and pregnancy: hormone and immune system interaction. International journal of dermatology. 1996;35(3):169-72.

18.Ceovic R, Mance M, Bukvic Mokos Z, Svetec M, Kostovic K, Stulhofer Buzina D. Psoriasis: female skin changes in various hormonal stages throughout life--puberty, pregnancy, and menopause. BioMed research international. 2013;2013:571912.

19.Black BP, Holditch-Davis D, Sandelowski M, Harris BG. Comparison of pregnancy symptoms of infertile and fertile couples. The Journal of perinatal \& neonatal nursing. 1995;9(2):1-9.

20.Dahiya K, Sharma D, Rajotia N, Jindal R. Herpes gestationis. Taiwanese journal of obstetrics \& gynecology. 2009;48(4):425-7.

21.Ambros-Rudolph CM. Dermatoses of pregnancy. Journal der Deutschen Dermatologischen Gesellschaft $=$ Journal of the German Society of Dermatology : JDDG. 2006;4(9):748-59; quiz 60-1.

22.Martin AG, Leal-Khouri S. Physiologic skin changes associated with pregnancy. International journal of dermatology. 1992;31(6):375-8.
23.Kumari R, Jaisankar TJ, Thappa DM. A clinical study of skin changes in pregnancy. Indian journal of dermatology, venereology and leprology. 2007;73(2):141.

24.Panicker VV, Riyaz N, Balachandran PK. A clinical study of cutaneous changes in pregnancy. Journal of epidemiology and global health. 2017;7(1):63-70.

25.Muzaffar F, Hussain I, Haroon TS. Physiologic skin changes during pregnancy: a study of 140 cases. International journal of dermatology. 1998;37(6):429-31.

26.Raj S, Khopkar U, Kapasi A, Wadhwa S. Skin in pregnancy. Indian journal of dermatology, venereology and leprology. 1992;58:84-8.

27.Kroumpouzos G, Cohen LM. Specific dermatoses of pregnancy: an evidence-based systematic review. American journal of obstetrics and gynecology. 2003;188(4):1083-92.

28.Bayerl C. [Acne therapy in pregnancy]. Der Hautarzt; Zeitschrift fur Dermatologie, Venerologie, und verwandte Gebiete. 2013;64(4):269-73.

29.de Haan TR, Beersma MF, Claas EC, Oepkes D, Kroes AC, Walther FJ. Parvovirus B19 infection in pregnancy studied by maternal viral load and immune responses. Fetal diagnosis and therapy. 2007;22(1):55-62.

30.Puri S, Pooni P, Mohan B, Bindal $\mathrm{V}$, Verma $S$, Verma $S$, et al. Pregnancy With SLE and Fetal Congenital Heart Block: A Case Report. Cardiology research. 2013;4(3):126-8. 
SOHAG MEDICAL JOURNAL

Vol. 22 No.3 October 2018
Cutaneous Changes During Pregnancy Heba Ahmad Anwar Mahmoud ${ }^{1}$ 